ARTICLE

\title{
Numerical Analysis on Thermal-Hydraulics of Supercritical Water Flowing in a Tight-Lattice Fuel Bundle
}

\author{
Toru NAKATSUKA*, Takeharu MISAWA, Hiroyuki YOSHIDA and Kazuyuki TAKASE \\ Japan Atomic Energy Agency, 2-4 Shirakata-Shirane, Tokai-mura, Naka-gun, Ibaraki-ken, 319-1195, Japan
}

\begin{abstract}
To evaluate thermal hydraulic characteristics of a tight-lattice fuel bundle of a supercritical water reactor (Super Fast Reactor), a simplified 19-rod fuel assembly was analyzed with a three-dimensional two-fluid model analysis code ACE-3D which has been enhanced by Japan Atomic Energy Agency. In ACE-3D, a two-phase flow turbulent model based on the k- $\varepsilon$ model was adopted. In this calculation, a one-twelfth model is adopted as the computational domain and takes advantage of symmetry. As boundary conditions, mass velocity, inlet enthalpy and power per rod are to be the same as the steady state condition of the Super Fast Reactor. Cross-sectional local power distribution in the fuel assembly is set to be flat. The effect of grid spacers is taken into account in the analysis. Calculated rod surface temperatures take peak values near the top of the rods. The maximum cladding surface temperature (MCST) is observed at the position facing the narrowest gap on the center rod near the outlet and the value is $901 \mathrm{~K}$ $\left(628^{\circ} \mathrm{C}\right)$. It was confirmed that the predicted MCST satisfies the thermal design criteria to ensure fuel and cladding integrity: the MCST should be less than $650^{\circ} \mathrm{C}$.
\end{abstract}

KEYWORDS: supercritical water reactors, supercritical fluid, heat transfer, fuel assemblies

\section{Introduction}

The supercritical-water-cooled reactor (SCWR) is a high-temperature, high-pressure water-cooled reactor that is operated above the critical pressure of water $(22.1 \mathrm{MPa})$. The concept aims at a high thermal efficiency and cost reduction based on decades of experience with conventional light water reactors (LWRs) and supercritical pressure fossil-fired power plants. The concept has been selected as a Generation IV nuclear energy system. In Japan, both thermal spectrum option (Super Light Water Reactor) and fast spectrum option (Super Fast Reactor) have been proposed. ${ }^{1)}$

In order to design the plant system or to evaluate the safety margin, it is important to assess thermal hydraulics in fuel assemblies in the core. As an analytical tool for that purpose, Japan Atomic Energy Agency (JAEA) has been enhancing a three-dimensional two-fluid model analysis code, ACE-3D, which was originally developed and applied for two-phase flow characteristics in LWRs, ${ }^{2}$ to predict thermal-hydraulic behavior of the SCWR. ${ }^{3)}$ The merit of using the two-fluid model is that the code can be used for not only normal operating condition, but also start-up, shut-down and transient conditions.

To evaluate prediction accuracy of the ACE-3D, three-dimensional numerical simulations had been performed based on several experimental data for tubes, single pins and bundle configurations. Numerical analysis results of heat transfer in a simplified 19-rod fuel assembly based on the design of the Super Fast Reactor concept was also ana-

*Corresponding author, E-mail:nakatsuka.toru@jaea.go.jp

(C) 2011 Atomic Energy Society of Japan, All Rights Reserved. lyzed and implied.

The present paper describes numerical simulation results of the simplified 19-rod fuel assembly considering grid spacer effects.

\section{Outline of ACE-3D Code}

The ACE-3D is a three-dimensional two-fluid model analysis code to simulate water-vapor or water-air two-phase flow in a rod bundle in subcritical pressure conditions. ACE-3D can also simulate single phase flow in supercritical pressure conditions.

In the two-fluid model, conservation equations of mass, momentum and energy for both liquid and vapor phases are calculated. Each phase is distinguished by the void fraction. However, the void fraction cannot be defined in supercritical pressure region where a phase change doesn't occur. Therefore, the actual saturation curve defined only in subcritical pressure region is extrapolated to the supercritical pressure region, so that the void fraction can be defined virtually even in the supercritical pressure region. ${ }^{4)}$

The ACE-3D adopts a two-phase flow turbulent model which is based on standard k- $\varepsilon$ model with a wall function. However, it was clarified by our previous study ${ }^{4}$ that the standard $\mathrm{k}-\varepsilon$ model underestimates wall temperature on heated walls because utilization of the wall function causes overestimation of turbulent eddy diffusivity of computational cells adjacent to the wall boundary. This implies that the wall treatment of the standard k- $\varepsilon$ model has a problem with estimating the turbulent heat flux on the wall boundary. Therefore, since in the succeeding study, the ACE-3D had 
adopted an equivalent eddy diffusivity model ${ }^{5)}$ for estimating turbulent eddy diffusivity in computational cells adjacent to a wall boundary. ${ }^{6}$ This model suppresses overestimation of turbulent eddy diffusivity at a wall boundary on the assumption that the amount of turbulent heat transport through the viscous sub-layer and that through the buffer layer should be equal. The Sommerfeld radiation condition was also introduced to improve analytical performance and stability of ACE-3D for supercritical pressure fluid. ${ }^{7)}$

\section{Calculation Model}

Our final goal is to evaluate thermal hydraulic characteristics of a real-sized fuel assembly. However, the present computational resource capability precludes the possibility of simulating a whole fuel bundle comprising around 270 fuel rods, even using a one-twelfth symmetry model. That led to the usage of a simplified fuel assembly model adopted in the present study.

The analytical geometry simulates a 19-rod fuel assembly, which is one of the most simplified geometries for the Super Fast Reactor fuel assembly in the sense that it includes three kinds of different sub-channels: (1) adjacent to the channel box; (2) next to type (1); and (3) located inside types (1) and (2). Figure 1 shows a cross section of the simplified fuel bundle. The rod diameter is $5.5 \mathrm{~mm}$, the gap between the rods is $1.045 \mathrm{~mm}$ and the axial length is $2.0 \mathrm{~m}$. The shroud wall is designed to distribute the coolant flow uniformly for each sub-channel.

One-twelfth of the area, the shaded section in Fig. 1, is adopted as the computational domain. The computational cell division and the boundary conditions are shown in Fig. 2. Computational mesh numbers of each block are shown in Table 1. Calculation conditions are chosen to be the same as the steady state of the Super Fast Reactor (Table 2). The cross-sectional local power distribution in the fuel assembly is set to be flat. Figure 3 shows the axial power profile of the fuel rods. To assess effects of grid spacers downstream along the axial direction, two grid spacers are installed at the locations that divide the heated portion into three equal lengths (Fig. 4).

\section{Calculation Results}

Figure 5 shows calculated rod surface temperature profile

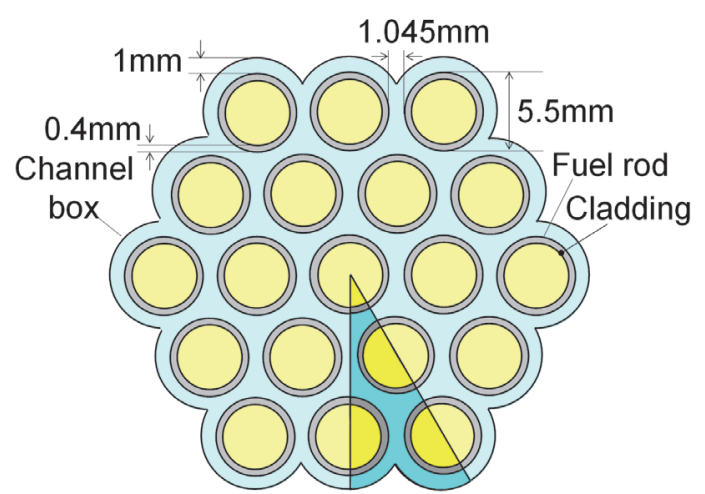

Fig. 4 Cross section of simplified fuel assembly

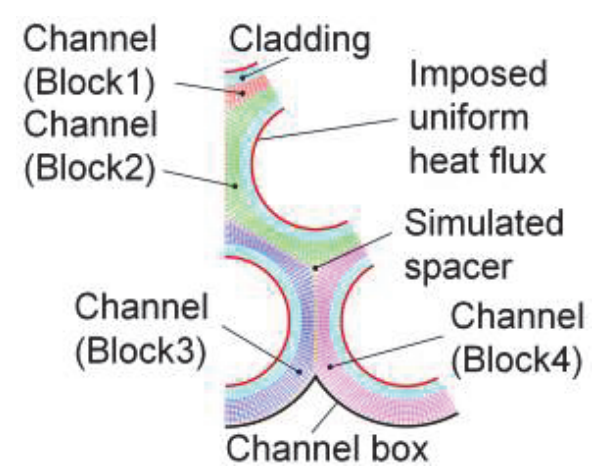

Fig. 1 Cell division and boundary

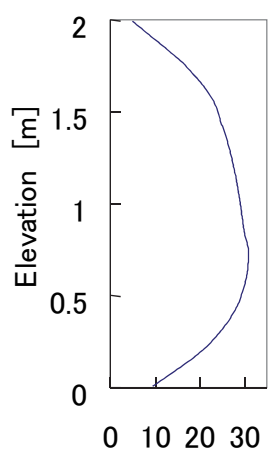

Linear heat generation rate $[\mathrm{W} / \mathrm{m}]$

Fig. 2 Axial power profile

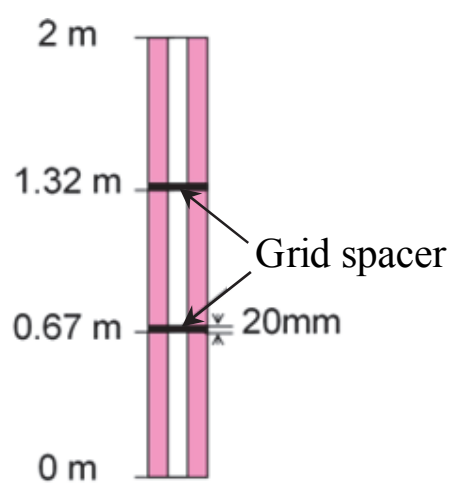

Fig. 3 Axial locations of grid spacers

Table 1 Computational mesh numbers

\begin{tabular}{ccccc}
\hline & Block & Block & Block & Block \\
& 1 & 2 & 3 & 4 \\
\hline Radial direction & \multicolumn{3}{c}{10 (channel), 5 (cladding) } \\
$\begin{array}{c}\text { Peripheral direc- } \\
\text { tion }\end{array}$ & 10 & 60 & 63 \\
Axial direction & \multicolumn{2}{c}{192} \\
\hline
\end{tabular}

Table 2 Calculation conditions

\begin{tabular}{ccc}
\hline $\begin{array}{c}\text { Mass velocity } \\
{\left[\mathrm{kg} / \mathrm{m}^{2} \mathrm{~s}\right]}\end{array}$ & $\begin{array}{c}\text { Inlet enthalpy } \\
{[\mathrm{kJ} / \mathrm{kg}]}\end{array}$ & $\begin{array}{c}\text { Power/rod } \\
{[\mathrm{kW}]}\end{array}$ \\
\hline 2406 & 2091 & 47 \\
\hline
\end{tabular}


along the fuel rods at four locations in the fuel assembly. Rod surface temperatures increase with the elevation and drop just downward of the spacer and increase again and take peak values near the top of the rods. The maximum cladding surface temperature (MCST) takes place at the position facing the narrowest gap on the center rod (P2 in Fig. 5) and are $901 \mathrm{~K}\left(628^{\circ} \mathrm{C}\right)$, which is almost the same as results without grid spacers and satisfies a principle of thermal design criteria to ensure fuel and cladding integrity: the MCST should be less than $650{ }^{\circ} \mathrm{C} .^{8)}$ Figure 6 shows the calculated horizontal temperature distribution in the fuel assembly. The temperatures are rather uniform except in the vicinity of channel box, which indicates that the wall configuration may be effective to flatten the coolant flow distribution among sub-channels in the fuel assembly. Figures 7 and 8 show the axial velocity distribution and the turbulent energy distribution, respectively. The effect of the grid spacers, such as a decrease in axial velocity and increase in turbulent energy at the positions of the spacers, are observed. The MCST could be reduced further by optimizing the number and configuration of grid spacers.

\section{Conclusion}

In the present study, thermal hydraulics of supercritical

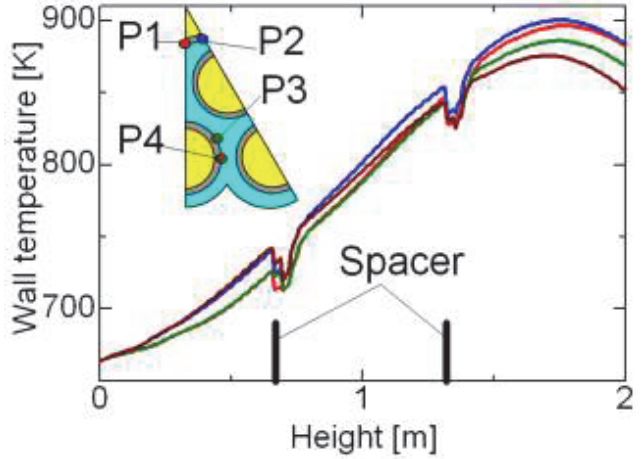

Fig. 5 Surface temperature profile along the fuel rods

pressure flow in rod bundle is assessed using a three-dimensional two-fluid model analysis code, ACE-3D., One-twelfth of a 19-rod fuel assembly with grid spacer effects, which simulates core flow in the fuel bundle of SCWR, is selected as the analytical domain. Calculated MCST satisfies thermal design criteria to ensure fuel and cladding integrity. The value can be lowered by optimizing the number and configuration of grid spacers.
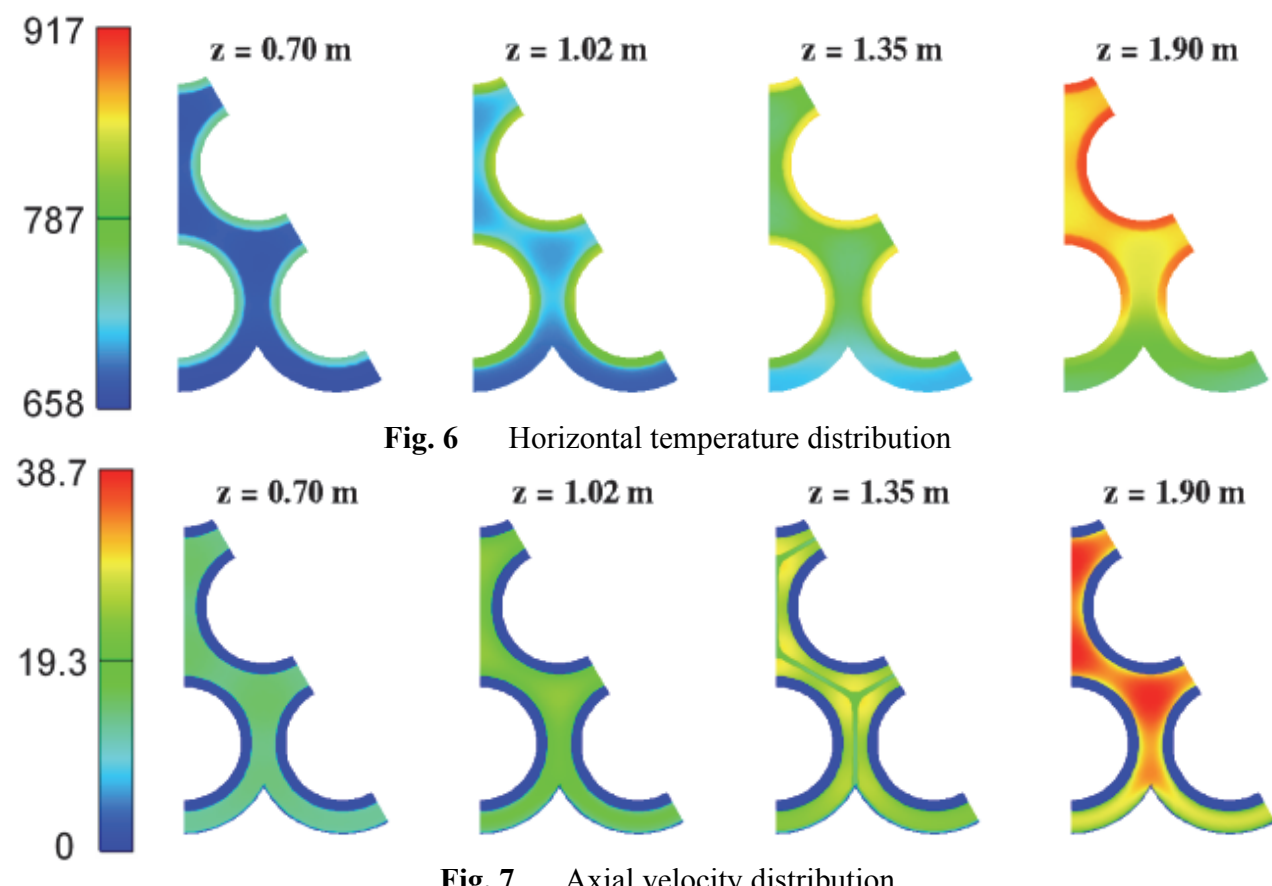

Fig. 6 Horizontal temperature distribution
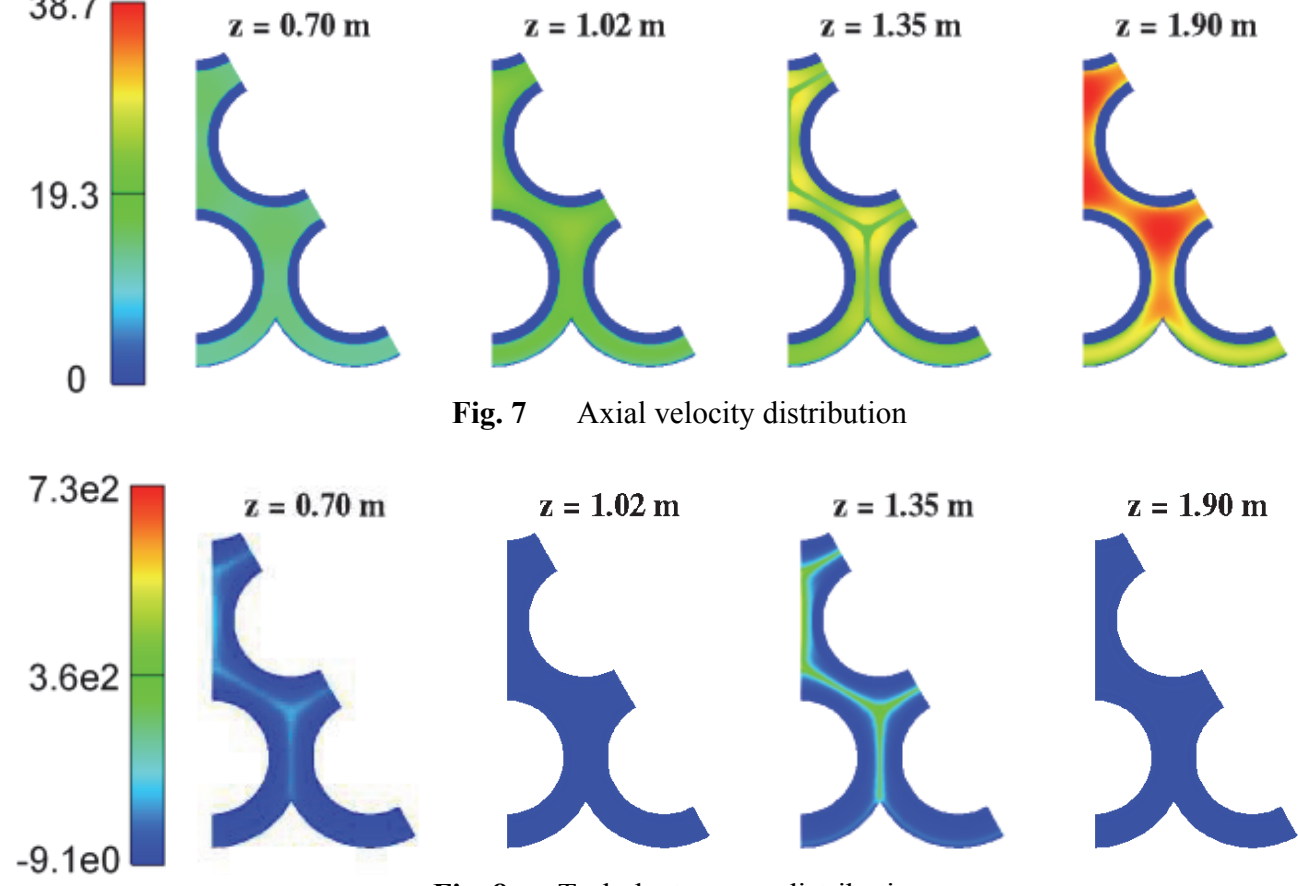

Fig. 7 Axial velocity distribution
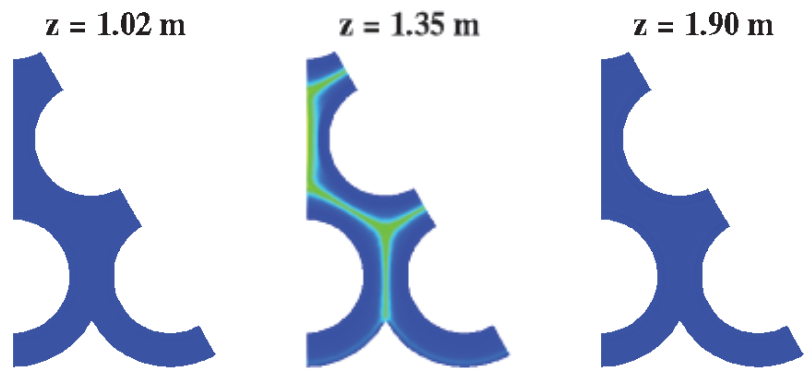

Fig. 8 Turbulent energy distribution 


\section{Acknowledgment}

The present study is the result of "Research and Development of the Super Fast Reactor" entrusted to The University of Tokyo by the Ministry of Education, Culture, Sports, Science and Technology of Japan (MEXT). This research was conducted using a supercomputer of Japan Atomic Energy Agency.

\section{References}

1) Y. Oka, S. Koshizuka, Y. Ishiwatari, A. Yamaji, Super Light Water Reactors and Super Fast Reactors, Springer, New York (2010).

2) A. Ohnuki, H. Kamo, H. Akimoto, Development of Multidimensional Two-Phase Model Code ACE-3D for Evaluation of Constitutive Equations, JAERI-Data/Code 96-033, Japan Atomic Energy Research Institute (JAERI) (1996), [in Japanese].

3) T. Misawa, H. Yoshida, H. Akimoto, "Development of Design Technology on Thermal-Performance in Tight-Lattice Rod Bundle: IV Large Paralleled Simulation by the Advanced Two-Fluid Model Code," J. Power Energy Syst., 2[1], 262-270
(2008).

4) T. Misawa, H. Yoshida, H. Tamai, K. Takase, "Numerical Analysis of Heat Transfer Test of Supercritical Water in a Tube Using the Three-Dimensional Two-Fluid Model Code," $J$. Power Energy Syst., 3 [1], 194-203 (2009).

5) T. Kunugi, H. Kawamura, "Application of a Two-Equation Turbulence Model to Heat Transfer and Fluid Flow of an Impinging Round Jet," Transport Phenomena in Turbulent Flows, Hemisphere Publishing Corp., New York, 593-604 (1988).

6) T. Misawa, T. Nakatsuka, H. Yoshida, K. Takase, "Numerical Analysis of Heat Transfer Experiment of Supercritical Pressure Water and Freon in a Rod bundle," Proc. 16th Pacific Basin Nuclear Conference (16PBNC), Aomori, Japan, Oct. 13-18, 2008, Paper ID P16P1065 (2008).

7) H. Yoshida, T. Nakatsuka, T. Suzuki, "Numerical Simulation of Heat Transfer Test of Forced Convection Supercritical Water in a Circular Pipe," Proc. 6th Japan-Korea Symposium on Nuclear Thermal Hydraulics and Safety (NTHAS6), Okinawa, Japan, Nov. 24-27, 2008, N6P1082 (2008), [USB memory].

8) L. Cao, Y. Oka, Y. Ishiwatari, Z. Shang, "Fuel, Core Design and Subchannel Analysis of a Superfast Reactor," J. Nucl. Sci. Technol., 45 [2], 138-148 (2008). 